\title{
Spatial Normalization of Human Back Images for Dermatological Studies
}

\author{
H. Mirzaalian ${ }^{1,3}$, G. Hamarneh ${ }^{1}$, T. K. Lee ${ }^{2,3}$ \\ ${ }^{1}$ Medical Image Analysis Lab at the School of Computing Science, Simon Fraser \\ University, BC, Canada, ${ }^{2}$ Cancer Control Research, BC Cancer Agency, BC Canada, \\ ${ }^{3}$ Photomedicine Institute, Department of Dermatology and Skin Science University of \\ British Columbia, and Vancouver Coastal Health Research Institute, BC, Canada
}

\begin{abstract}
Density of pigmented skin lesions (PSL) is a strong predictor of malignant melanoma. Some dermatologists advocate periodic fullbody scan for high-risk patients. It is clinically important to compare and detect changes in the number and appearance of PSL across time. However, manual inspection and matching of PSL is tedious, error-prone, and suffers from inter-rater variability. Therefore, an automatic method for tracking corresponding PSL would have significant health benefits. In order to automate the tracking of PSL in human back images, we must perform spatial normalization of the coordinates of each PSL, as is done in brain atlases. We propose the first human back template (atlas) to obtain this normalization. Four pairs of anatomically meaningful landmarks (neck, shoulder, armpit and hip points) are used as reference points on the skin-back image. Using the landmarks, a grid with longitudes and latitudes is constructed and overlaid on each subject specific back image. To perform spatial normalization, the grid is registered into the skin-back template, a unit-square rectilinear grid. We demonstrate the benefits of our approach, on 56 pairs of real dermatological images, through the increased accuracy of PSL matching algorithms when our anatomy-based normalized coordinates are used.
\end{abstract}

\section{Introduction}

Melanoma is one of the fastest growing cancers among the white population in the world with an average $3 \%$ increase in incidence for the last four decades. In the USA and Canada alone, it was estimated that there will be 73,720 cases of melanoma in 2009 [2,3]. The mechanism of melanoma development is not fully understood. Nevertheless, pigmented skin lesions (PSL) density (number of PSL per unit area of skin) have been reported as the strongest risk factor, with about $50 \%$ of melanoma originating from pre-existing PSL. Early diagnosis of melanoma may lead to potentially life-saving therapy. To allow for early diagnosis, patients are full-body scanned periodically and digital color two dimensional images of the skin are collected during the process. One example of back images at two different times is shown in Figure 1. During a dermatological examination, physicians compare the skin images at different time instances to 
observe changes in the number of PSL and their appearance, such as their size, color, shape, and texture. However, relying on visual inspection of PSL is costly, time consuming, and may be error prone due to user fatigue [11]. Therefore, an automatic method for matching corresponding PSL would have significant health benefits, especially for tracking PSL in patients who are at a high risk of developing melanoma and, hence, require regular PSL examinations.

In order to have an anatomically meaningful comparison between the PSL in the human back images, we proposed to transform the back images into a common normalized space [14]. This approach was inspired by the works on human brain atlases, which have benefited the medical image analysis research in many ways; mainly by providing a common frame of reference that facilitates crosssectional and longitudinal comparisons of the brain anatomy $[17,18,8,13,10]$. In this paper, we focus on the construction of the first human back atlas.

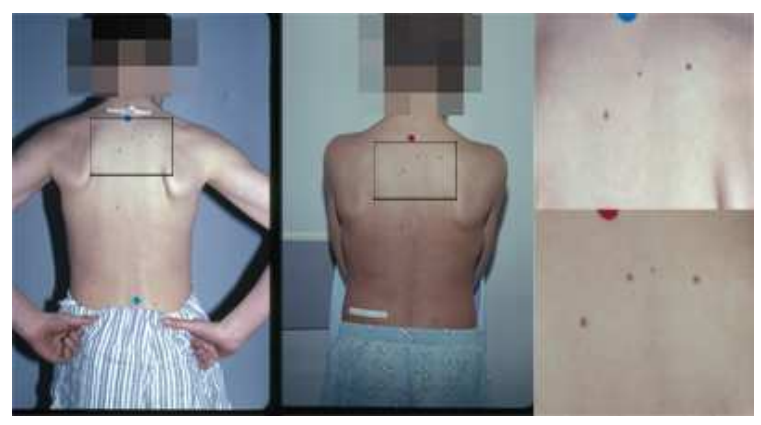

(a)

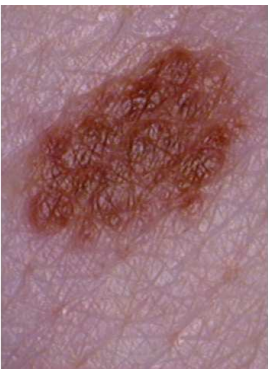

(b)

Fig. 1: (a) Constellation of PSL on a back image [9], (b) A close up on a big PSL using a dermoscope with non-polarized light.

\section{Proposed Back Template}

\subsection{Coordinate System}

The proposed template is a unit-square patch ${ }^{1}$ with domain $\Omega \subset[-0.5,0.5] \times$ $[-0.7,0.3]$ as shown in Figure 2(b). The template is constructed as follows. Four pairs of points corresponding to anatomically meaningful landmarks (i.e. Type $I$ and $I I$ as defined in $[1,7]$ ) are used as reference points on the template (Figure $2(\mathrm{a}))$ :

\footnotetext{
${ }^{1}$ Without loss of generality we choose unit patch square template instead of rectangle, since the square and rectangle are linearly transformable to each other, i.e. by performing anisotropic scaling.
} 
i. The point where the left (right) side of the neck meets the left (right) shoulder, or neck-left $n_{l}$ (neck-right $n_{r}$ ) for short, corresponds to $N_{l}\left(N_{r}\right)$ in the normalized space of the template. Note that we use capital letters for template and small letters for image space coordinates.

ii. The point where the left (right) shoulder meets the left (right) arm, denoted by shoulder-left $s_{l}$ (shoulder-right $s_{r}$ ), corresponds to $S_{l}=$ $(-0.5,0.3)\left(S_{r}=(0.5,0.3)\right)$ in the normalized space of the template.

iii. The left and right armpits $\left(a_{l}\right.$ and $a_{r}$, respectively) correspond to $A_{l}=$ $(-0.5,0)$ and $A_{r}=(0.5,0)$ in the normalized space of the template, respectively.

iv. The left hip point $\left(h_{l}\right)$ and right hip point $\left(h_{r}\right)$ correspond to $H_{l}=$ $(-0.5,-0.7)$ and $H_{r}=(0.5,-0.7)$ in the normalized space of the template, respectively.

According to the classification of the landmarks by Bookstein [1,7], Type $I$ landmark is defined as the discrete juxtapositions of tissues e.g. the point where three structures meet, the branching point of the tree structures, or the intersections of extended curves with planes of symmetry. The armpit point is the lateral intersection of left (right) arm and the backs left (right) silhouette and hence, can be classified as Type I. Landmark Type II is defined as the maximum of curvature or other local morphogenetic process. The neck and shoulder points are of this type.

Note that all the anatomical landmark locations in the template space (except $N_{l}$ and $N_{r}$ ) are at specific coordinates in the domain $\Omega$ (Figure 2(b)). The subject specific landmarks, on the other hand, can be any spatial coordinates in the physical image space. The coordinate system of the template is formed by longitudes and latitudes (Figure 2(b)).

The central latitude is a straight horizontal line segment $\overline{A_{l} A_{r}}$ which connects $A_{l}$ and $A_{r}$, whereas the central longitude is the line segment $\overline{N_{m} H_{m}}$ connecting the medial point $N_{m}=(0,0.3)$ with $H_{m}=(0,-0.7)$ and passing through $S_{m}=$ $(0,0.3)$ and $A_{m}=(0,0)$, where the subscript $m$ indicates the midpoint between the corresponding left and right reference points, e.g. $N_{m}=\left(N_{l}+N_{r}\right) / 2$. The topmost (superior) latitude $\overline{S_{l} S_{r}}$, the bottommost (inferior) latitude $\overline{H_{l} H_{r}}$, the leftmost longitude $\overline{S_{l} H_{l}}$, and the rightmost longitude $\overline{S_{r} H_{r}}$ define the borders of the domain $\Omega$ of the template. Note that in the template space: $\overline{A_{l} A_{r}} \perp \overline{N_{m} H_{m}}$, $\overline{S_{l} H_{l}}\left\|\overline{N_{m} H_{m}}\right\| \overline{S_{r} H_{r}}$ and $\overline{S_{l} S_{r}}\left\|\overline{A_{l} A_{r}}\right\| \overline{H_{l} H_{r}}$ where $\perp$ denotes perpendicular and $\|$ denotes parallel. Finally, based on the anatomical landmarks and the reference central latitude and longitude, a template based rectilinear coordinate system is defined and a complete Cartesian grid is overlaid on the template. 


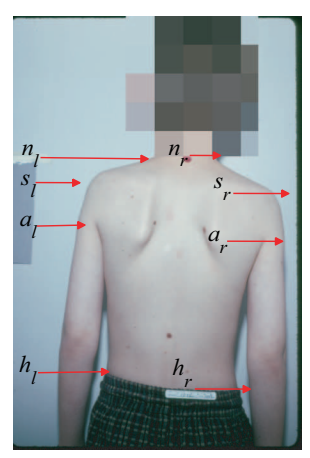

(a) Landmark

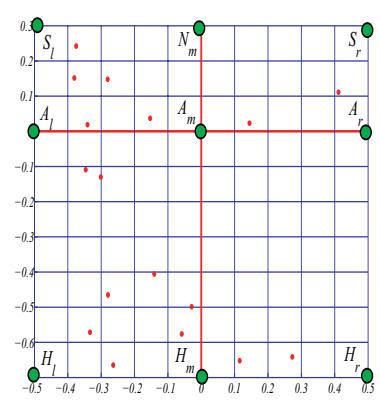

(b) Template

Fig. 2: Human back template. (a) Landmarks are shown in the image. (b) Template of the back image. The red and green points correspond to the normalized coordinates of the PSL and the reference anatomical landmarks, respectively. The vertical and horizontal red lines correspond to the central longitude and latitude.

\subsection{Spatial Normalization of Back Coordinates}

At a high level, the spatial normalization is done by following these steps: detecting the basic longitudes and latitudes in the image space, constructing the grid, and establishing a mapping between the continuous image space and the template. The details are described below.

Detecting the Basic Longitudes and Latitudes To perform spatial normalization, a set of six basic latitudes (Superior, Central, and Inferior) and longitudes (Left, Right and Central) is overlaid on each subject specific back image to register it into the template coordinate system (Figure 3). The latitudes and longitudes are constructed using the aforementioned anatomical landmark points, some lateral edge points of the left and right silhouette of the back, and superior edge points of both shoulders' silhouettes (Figure 3(a)). Note that, most of the latitudes and longitudes in the image space are smooth curves fitted to the anatomical landmarks and medial lines as explained below. Consequently, the grid in the image space is no longer rectilinear but rather curved. We now provide the details on how the latitudes and longitudes are constructed.

i. The Left (Right) longitude as shown in Figure 3(b) is a degree 3 polynomial least-squares fitted to the lateral edge points of the back's left (right) silhouette and constrained to pass through $a_{l}\left(a_{r}\right)$. Least square fitting calculates the polynomial coefficient such that the polynomial passes as close as possible to the points, minimizing the square of the error distance. We use a degree 3 polynomial because it provides sufficient degrees of freedom to model the sides without excessive inflection points. Degree 2 is not flexible enough and higher degree than 3 would 
increase the complexity in addition to over-fitting the curve to the noise in the detected edge points.

ii. The Central longitude (Figure 3(c)) is the medial curve between the left and right longitudes and is calculated as follows: First, we calculate the distance transform $(D T)$ between the Left and Right longitudes. Next, we calculate the gradient of the $D T$ image: $\nabla(D T)$, which captures the amount by which the $D T$ image changes along the vertical and horizontal directions. Finally, we construct the center longitude via a degree 3 polynomial least-squares fitted to the local maxima of the gradient magnitude of $D T:|\nabla(D T)|$ (Figure 4). Alternatively, we can calculate the center longitude as the loci of points midpoint between the Left and Right longitudes (Figure 4). We obtained similar results using both approaches. The second definition, however, is preferable because its simpler to compute, whereas the first is more rigourous mathematically.

iii. Superior latitude (Figure $3(\mathrm{~d})$ ) is a degree 6 polynomial least-squares fitted to the superior edge points of both shoulders'silhouettes. Degree 6 is chosen to accommodate the number of concavities and convexities that can occur along these superior edge points.

iv. Central latitude (Figure $3(\mathrm{e})$ ) is a straight line segment $\overline{a_{l} a_{r}}$ connecting $a_{l}$ to $a_{r}$.

v. Inferior latitude (Figure $3(\mathrm{f})$ ) is a straight line segment $\overline{h_{l} h_{r}}$ connecting $h_{l}$ and $h_{r}$.

As a result, the image domain of a specific subject's back, $\omega \subset R^{2}$, is bounded by the left and right longitudes and superior and inferior latitudes.

Constructing the Grid The intersections of the basic longitudes and latitudes can be considered as the control points for the mapping between two spaces. As more control points increase the precision of the mapping, we interpolate as many additional longitudes and latitudes to achieve a desired accuracy. The lines are interpolated between the basic longitudes and attitudes using equal arc length sampling points, which can be classified as Type III landmarks [1]. Figure $3(\mathrm{~g})-3(\mathrm{l})$ shows the steps of interpolating longitudes and latitudes of the grid

Mapping the Continuous Spaces After established the control points, we set up the correspondence between any point in the two continuous domains (not only the landmarks, points on the latitudes and longitudes or their intersections) by warping the grid to the template. We evaluated two interpolation methods: Barycentric coordinates (BC) [6] and Thin plate splines (TPS) [5].

Barycentric Coordinates (BC) Method: The BC method can map points using the following steps: 


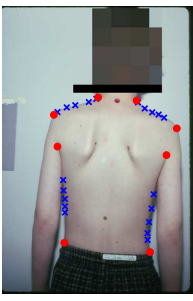

(a)

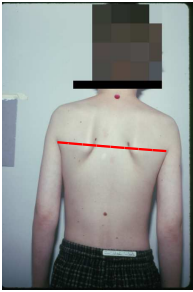

(e)

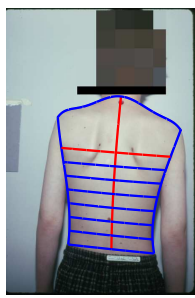

(i)

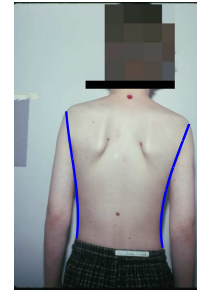

(b)

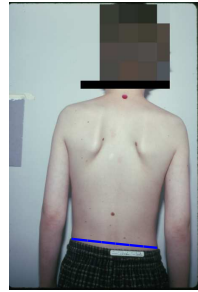

(f)

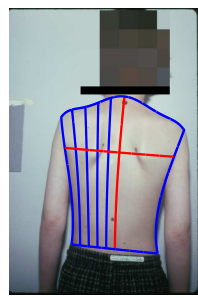

(j)

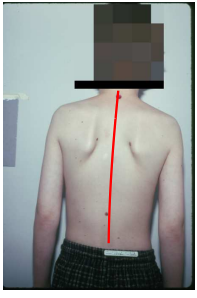

(c)

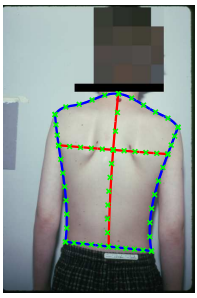

$(\mathrm{g})$

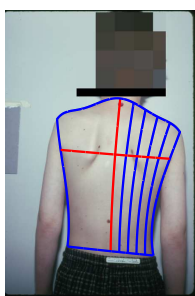

(k)

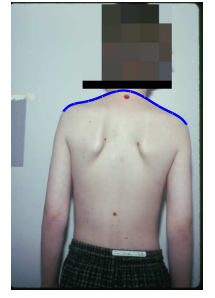

(d)

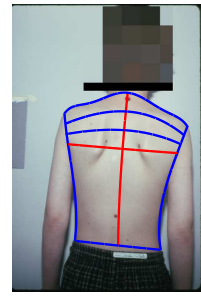

(h)

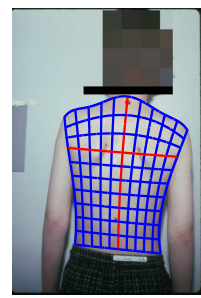

(1)

Fig. 3: Different steps of the grid construction: (a) Points inserted by the user (blue are the edge points, and red are the landmarks) (b) Left and Right longitudes, (c) Central longitude, (d) Superior latitude, (e) Central latitude, (f) Inferior latitude, (g) Equal arc length samplings , (h) Interpolating latitudes between superior and central latitudes, (i) Interpolating latitudes between central and inferior latitudes, (j) Interpolating longitudes between left and central longitudes, (k) Interpolating longitudes between right and central longitudes, (l)The grid overlaid on the back image.

i. For each point, $p=\left(p_{x}, p_{y}\right) \in \omega$, find the cell $\operatorname{cell}(p) \subset \omega$ containing $p$, i.e. $\operatorname{cell}(p)$ is the area enclosed between the two nearest longitudes and latitudes to $p$. As shown in Figure 5(a)-5(b), we locate the points $a=\left(a_{x}, a_{y}\right), b=\left(b_{x}, b_{y}\right)$, and $c=\left(c_{x}, c_{y}\right)$.

ii. Find the corresponding cell $C E L L(P) \subset \Omega$ in the template domain. $C E L L(P)$ is the area enclosed by the corresponding longitudes and lat- 


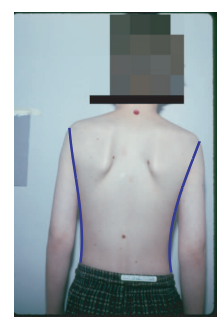

(a)

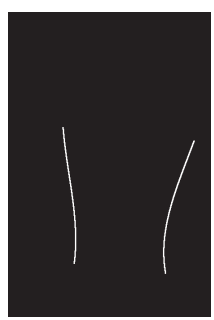

(b)

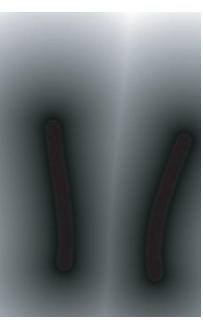

(c)

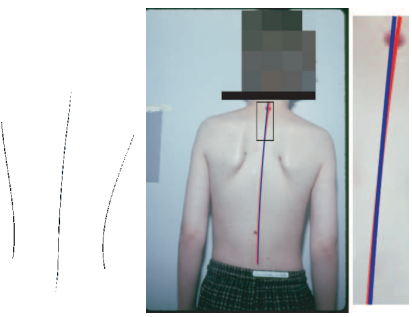

(d)

(e)

Fig. 4: (a) Image contains the left and right longitudes. (b) Binary image contains the left and right longitudes. (c) The distance transform of the image (b). (d) Points with maximum gradient. (e) Red and blue curves show the central longitude resulted from $D T$ and average line between the left and right longitudes respectively. A close up of the small rectangular region near the neck is shown on the right.

itudes of the bounding $\operatorname{cell}(p)$ in the image domain. For example, we locate the control points $A, B$ and $C$ as shown in Figures 5(c)-5(d).

iii. Compute the BC coordinates $\left(t_{1}, t_{2}, t_{3}\right)$ of $p$ using (1) [6].

$$
\left|\begin{array}{c}
t_{1} \\
t_{2} \\
t_{3}
\end{array}\right|=\left|\begin{array}{ccc}
a_{x} & b_{x} & c_{x} \\
a_{y} & b_{y} & c_{y} \\
1 & 1 & 1
\end{array}\right|^{-1}\left|\begin{array}{c}
p_{x} \\
p_{y} \\
1
\end{array}\right|
$$

iv. The point $P \subset \Omega$ is determined by the same $\mathrm{BC}$ coordinates as $P=$ $t_{1} A+t_{2} B+t_{3} C$.

Thin Plate Splines (TPS) Method: TPS wraps points from one domain to another domain by mimicking the deformation of a thin-plate and minimizes the bending energy. In particular, interpolated coordinates using TPS are given by [5]:

$$
f(x, y)=\sum_{i=1}^{K} c_{i} \phi\left(\left|(x, y)-\left(x_{i}, y_{i}\right)\right|\right)+a_{10} x+a_{01} y+a_{00}
$$

where |.| denotes the usual Euclidian distance, $\left(x_{i}, y_{i}\right)$ is a set of control points, $a_{i j}$ and $c_{i}$ are warping coefficients representing the affine transformation and non-affine deformations, respectively. $\phi$ is referred as the Kernel function of the thin plate spline and is given by $\phi=r^{2} \log r$ where $r$ is the distance $\sqrt{x^{2}+y^{2}}$. The kernel models elasticity and non-rigid transformation and govern the displacement of the control points.

Our control points are intersections of the longitudes and latitudes. TPS fits a function $f$ such that any point $\left(x_{i}, y_{i}\right) \subset \omega$ is mapped to $\left(X_{i}, Y_{i}\right) \subset \Omega\left(\left(X_{i}, Y_{i}\right)\right.$ : $\left.\left(X_{i}, Y_{i}\right)=f\left(x_{i}, y_{i}\right)\right)$ by minimizing the following energy function [5]:

$$
\left.E=\iint\left(E_{b}(x, y)\right)^{2}\right) d x d y
$$


where $E_{b}(x, y)=\left(\frac{d^{2} f}{d x^{2}}\right)^{2}+2\left(\frac{d^{2} f}{d x d y}\right)^{2}+\left(\frac{d^{2} f}{d y^{2}}\right)$ is a measure of the bending energy at $(x, y)$.

The result of these two alternative interpolation methods is to stablish a bijective function $f: \omega \rightarrow \Omega$ mapping between points between two domains. In the BC method, curves bounding the cells are approximated by straight lines when establishing correspondence between the two continues domains. TPS does not rely on this assumption and hence it is more accurate. However, TPS is more computationally expensive than the BC. Using our database of back images described in section 3, the difference between the normalized coordinates using $\mathrm{BC}$ and TPS in the unit square patch is: $\left|f^{B C}(x, y)-f^{T P S}(x, y)\right|=2.3 \times$ $10^{-5} \pm 1.87 \times 10^{-7}$, which shows that the increase in accuracy does not justify the additional computational complexity of TPS.

Figure 6 demonstrates the mapping between the two spaces. Cells, longitudes and latitudes in the image and template space are colored with the same colors.

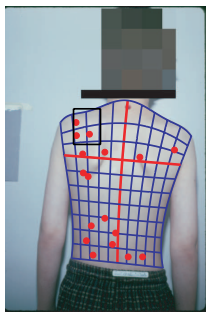

(a)

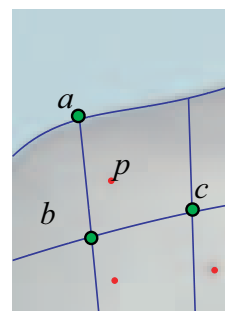

(b)

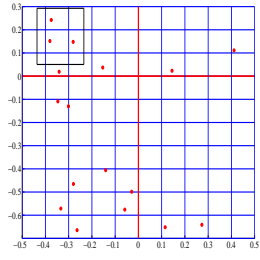

(c)

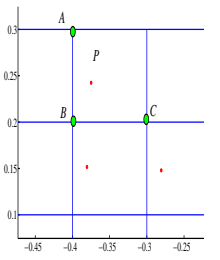

(d)

Fig. 5: Interpolating points by Barycentric Coordinates: (a) Points in the image domain. (b) Shows a close up of $\operatorname{cell}(p)$ which $p \in \operatorname{cell}(p)$. (c) Points in the template domain. (d) Shows a close up of $C E L L(P)$ correspond to $\operatorname{cell}(p)$.

\section{Back Images}

The data used in this study were obtained from color slides taken from an epidemiologic study concerning the use of broad-spectrum sunscreen and PSL development. The images were digitized with 24-bit color at $2000 \mathrm{dpi}$, with a final resolution of about $0.25 \mathrm{~mm} /$ pixel. A set of 56 pairs of digitized images containing PSL was chosen to evaluate the proposed method. Each image pair belongs to a single subject imaged at baseline and 3 years later. Ground truth identification of all PSL, correspondence of the PSL across the pair of images, as well as the anatomical landmarks were provided for images pairs by an expert user [9]. 


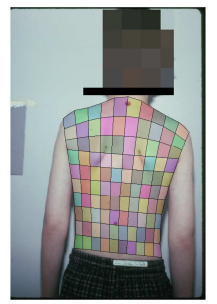

(a)

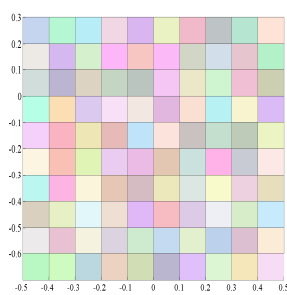

(b)

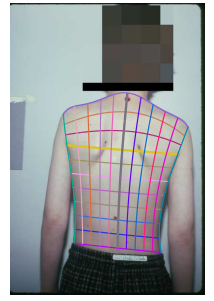

(c)

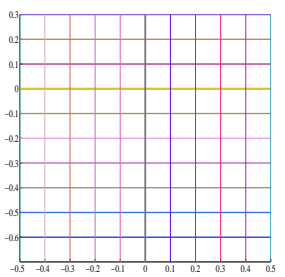

(d)

Fig. 6: Corresponding cells, longitudes and latitudes in the image and template domain are shown with the same color within the first and second rows respectively.

\section{Experiment}

Figure 7 shows four examples of the overlaid grids on the back images and the normalized coordinates of the PSL resulting from our proposed template. Corresponding latitudes and longitudes on the image-template space are shown with the same color. Our primary application of using normalized spatial coordinates is to match PSL between two images captured at different times. In order to show that utilizing normalized spatial coordinates improves the PSL matching, in [15], we compared the matching results of different algorithms with and without spatial normalization. The following matching algorithms were tested on the 56 pairs of dermatological images: Coherent Point Drift (CPD) [16], shape contexts (SC+TPS) [4], the spectral technique of (Spect) [12] and Hypergraph matching $(d=2)$ (Hyp) [20]. Examples of finding corresponding PSL automatically in real image data are shown in Figure 8 (for further details, we refer the reader to $[15])$.

Figure 9 shows the number of incorrect matches (NIM) for the state of the art matching algorithms. Blue and red bars show the error results using image space coordinates (no spatial normalization) versus using normalized space coordinates (using the proposed template), respectively. As it can be seen, using our proposed anatomy based normalized coordinates, we substantially improved the PSL matching accuracy for all algorithms.

\section{Conclusions}

An automatic method for matching PSL on human back images is of utmost importance for early detection of potential malignancies. In order to have an anatomically meaningful comparison between PSL in the human back images, we need to perform spatial normalization. In this work, we propose the first human back template to perform spatial normalization. The results show that using our proposed anatomy based normalized coordinates for the state of the 

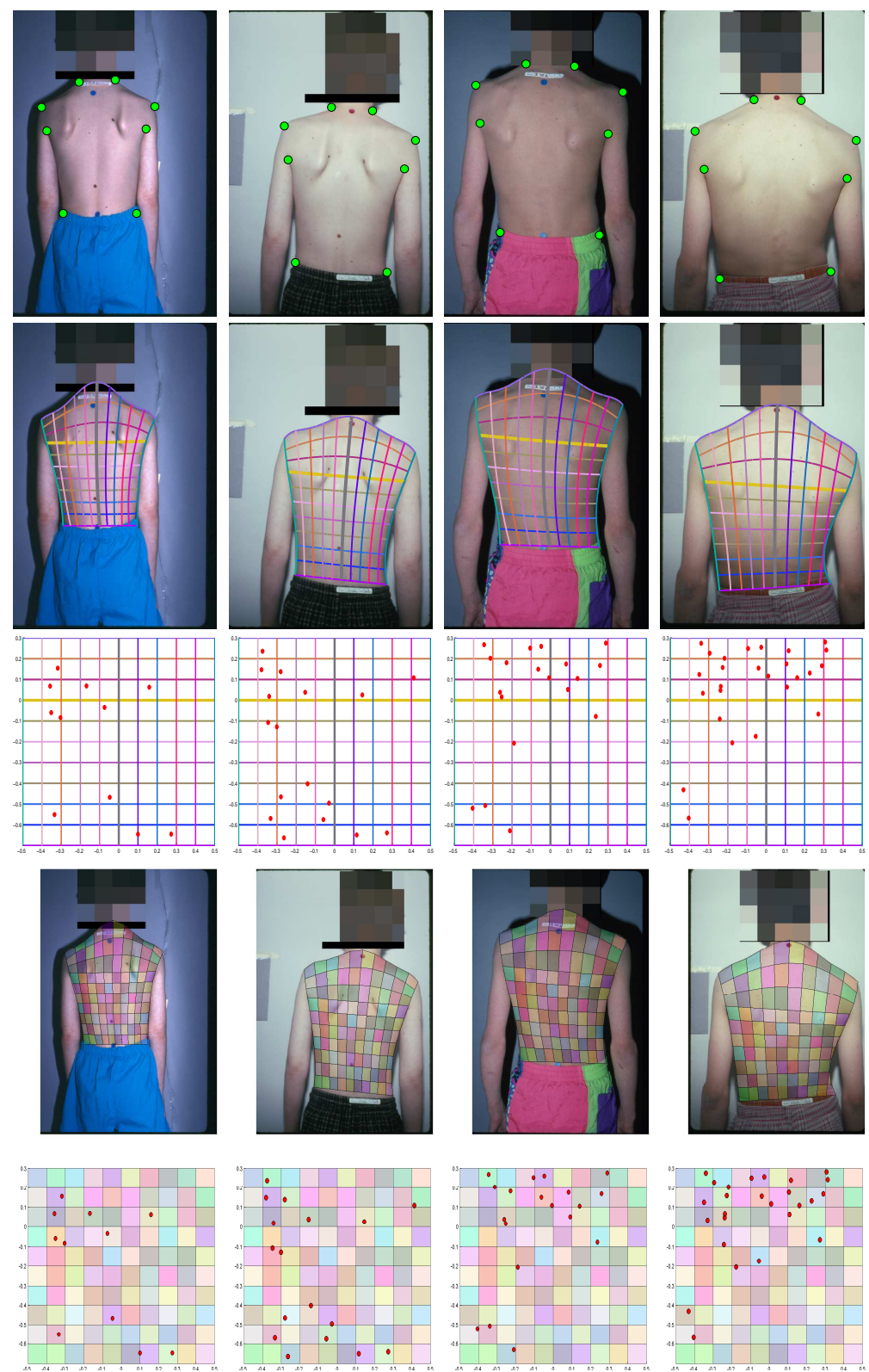

Fig. 7: Some example of the overlaid grid and the template. (a) The landmarks used in constructing the grid. (b) The overlaid grid on the back images. (c) The resulting normalized coordinates of the PSL using the proposed template. Corresponding latitudes and longitudes on the grid and the template are shown with the same color in (b-c). (d-e) Corresponding cells on the grid and the template are shown with the same color. Red points are correspond to the positions of the PSL. 

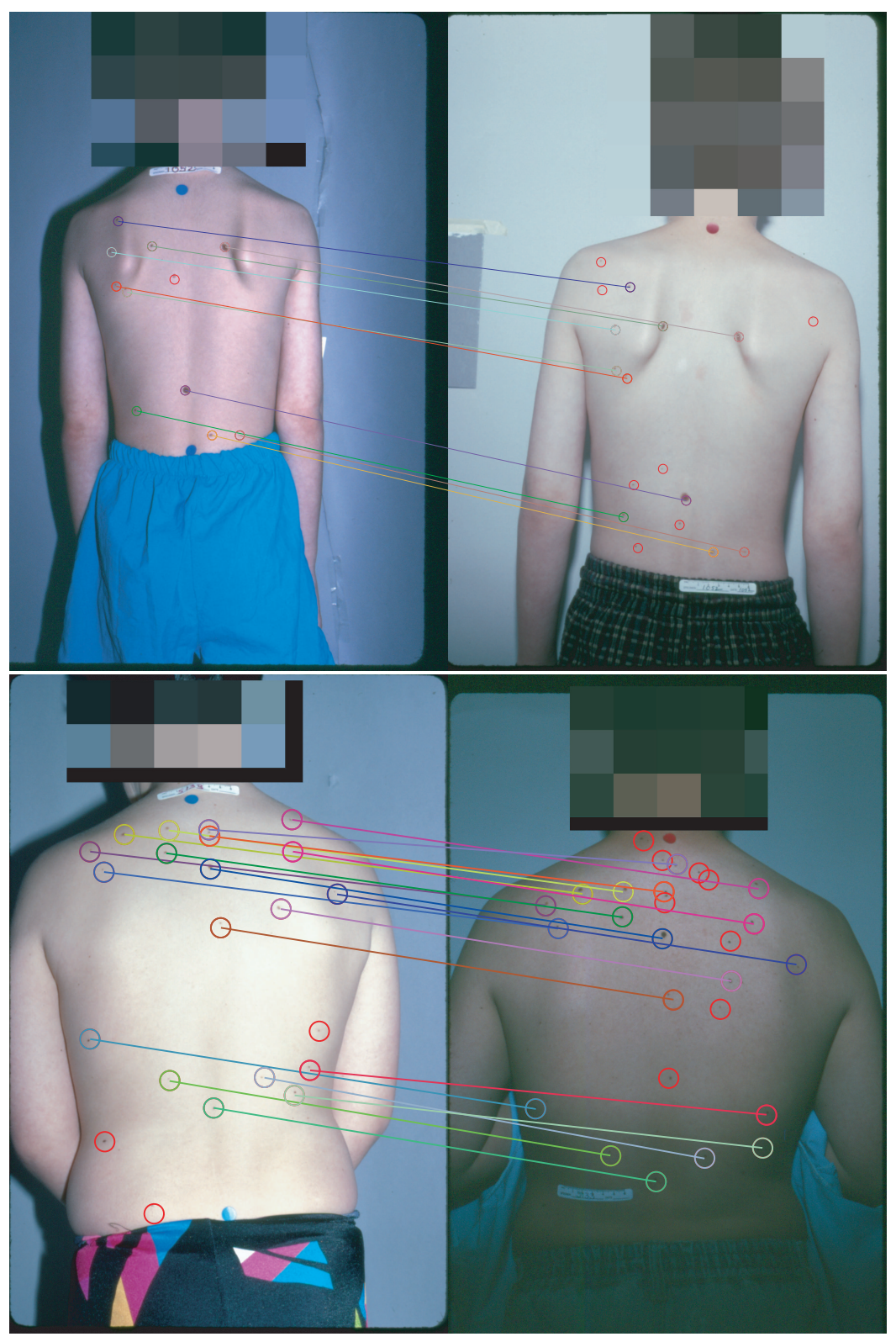

Fig. 8: Each row is a different subject. The images in the right column were captured 3 years later than the left column. The resulting PSL matching is indicated by lines. 


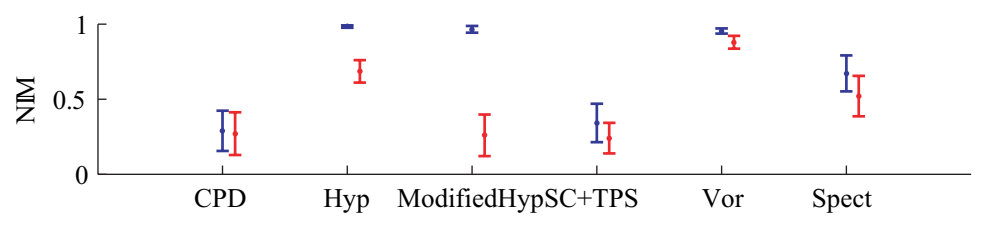

Fig. 9: PSL matching evaluation (using number of incorrect matches (NIM)) of different methods (along the horizontal axis) when spatial image coordinates (blue bars) or normalized coordinates (red bars) are used in the matching. We note a substantial improvement in accuracy when normalized coordinates are used.

art matching algorithms, we substantially improve the PSL matching accuracy. We do not claim that our template is the best possible template, so we plan to continue exploring alternative approaches to improve it and we anticipate other groups will do too. One of the challenges, as observed in this study, will be to develop a robust definition of the hip anatomical landmark, which we plan to address through discussions and consultations with dermatologists. We are also working on automated image processing methods for identifying the PSL and the anatomical landmarks, which will complement our work on PSL matching [15] and spatial normalization presented in this paper, in order to produce an endto-end system for reading pairs of human back images, counting the PSL, and detecting appearing and disappearing PSL. Suspicious PSL are further analyzed using shape and color and texture properties as in [19].

\section{References}

1. Morphometric Tools for Landmark Data. UK: Cambridge University Press, 1997. $2,3,5$

2. American cancer society (2009). cancer facts and figures 2009, american cancer society. 2009. Atlanta, USA. 1

3. Canadian cancer societys steering committee (2009). canadian cancer statistics 2009. 2009. Toronto, Canada. 1

4. S. Belongie, J. Malik, and J. Puzicha. Shape matching and object recognition using shape contexts. IEEE PAMI, 24(4):509-522, 2002. 9

5. F. Bookstein. Principal warps: thin-plate splines and the decomposition of deformations. IEEE PAMI, 11(6):567-585, 1989. 5, 7

6. O. Bottema. On the area of a triangle in barycentric coordinates. 8:228-231, 1982 . 5,7

7. T. Cootes, C. Taylor, D. Cooper, and J. Graham. Active shape models: Their training and application. 61(1):38-59, 1995. 2, 3

8. A. Evans, M. Kamber, D. Collins, and D. MacDonald. An MRI-based probabilistic atlas of neuroanatomy, chapter MRI-based probabilistic atlas of neuroanatomy, pages 263-274. Plenum Press, 1994. 2

9. R. Gallagher, J. Rivers, T. Lee, C. Bajdik, D. I. McLean, and A. Coldman. Broad-Spectrum Sunscreen Use and the Development of New Nevi in White Chil- 
dren: A Randomized Controlled Trial. Journal of American Medical Association, 283(22):2955-2960, 2000. 2, 8

10. D. Hill, P. Batchelor, M. Holden, and D. Hawkes. Medical image registration. Physics in medicine and biology, 46:R1-R45, 2001. 2

11. E. A. Holly, J. W. Kelly, S. N. Shpall, and S. H. Chiu. Number of melanocytic nevi as a major risk factor for malignant melanoma. J. Am. Acad. Dermatol, 17:459-468, 1987. 2

12. M. Leordeanu and M. Hebert. A spectral technique for correspondence problems using pairwise constraints. IEEE ICCV, 2:1482-1489, 2005. 9

13. J. Mazziotta, A. Toga, A. Evans, P. Fox, and J. Lancaster. A probabilistic atlas of the human brain: Theory and rationale for its development : The international consortium for brain mapping (icbm). NeuroImage, 2(Part 1):89-101, 1995. 2

14. H. Mirzaalian, G. Hamarneh, and T. Lee. Probabilistic graph for skin mole matching. Submitted for IEEE journal. 2

15. H. Mirzaalian, G. Hamarneh, and T. Lee. Graph-based approach to skin mole matching incorporating template-normalized coordinates. In IEEE CVPR, pages 2152-2159, 2009. 9, 12

16. A. Myronenko, X. Song, and M. Carreira-Perpinan. Non-rigid point set registration: Coherent point drift. In B. Schölkopf, J. Platt, and T. Hoffman, editors, NIPS, pages 1009-1016. MIT Press, 2007. 9

17. J. Talairach and P. Tournoux. Co-Planar Stereotaxic Atlas of the Human Brain: 3-Dimensional Proportional System : An Approach to Cerebral Imaging. Thieme Medical Publishers, 1988. 2

18. L. Thurfjell, C. Bohm, and E. Bengtsson. Cba-an atlas-based software tool used to facilitate the interpretation of neuroimaging data. Computer Methods and Programs in Biomedicine, 47(1):51 - 71, 1995. 2

19. P. Wighton, M. Sadeghi, and T. L. ans S. Atkins. A fully automatic random walker segmentation for skin lesions in a supervised setting. 5762:1108-1115, 2009. 12

20. R. Zass and A. Shashua. Probabilistic graph and hypergraph matching. IEEE CVPR, pages 1-8, 2008. 9 